\title{
FIRST YEAR EXPERIENCE OF GERMLINE MUTATION TESTING IN GYNECOLOGICAL CANCER PATIENTS IN A CLINICAL SETTING IN THE BAHAMAS
}

\section{S. Bowe ${ }^{1}$, R. Butler ${ }^{1}$, D. Halliday ${ }^{1}$, G. Rangel', D. Cerbon², R. Roberts ${ }^{1,3}$, B. Slomovitz ${ }^{2}$, M. Schlumbrecht ${ }^{2}$, S. George $^{2}$.}

1 University of the West Indies Clinical Medicine and Research, Department of Obstetrics and Gynecology, Nassau,

Bahamas; '2University of Miami- Miller School of Medicine, Department of Obstetrics-

Gynecology and Reproductive Sciences- Division of Gynecologic Oncology-Sylvester Comprehensive Cancer Center,

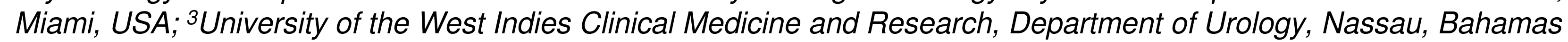

Objective: To facilitate genetic testing and determine the rate of hereditary ovarian cancer in The Bahamas we established an Oncology-led point of genetic testing at the Princess Margaret Hospital (PMH) in The Bahamas.

Introduction: BRCA1 and BRCA2 are multifunctional proteins that are associated with hereditary breast and ovarian cancer. Studies report that $65-85 \%$ of hereditary ovarian cancers are the result of $B R C A 1$ or $B R C A 2$ mutation (1). The lifetime risk of developing ovarian cancer in persons with BRCA1 or BRCA2 mutation is $20-40 \%$ compared with $1-2 \%$ in non-affected individuals. The Bahamas has a high rate of ovarian cancer and high rate of breast cancer. At least 1 in 5 women diagnosed with breast cancer have a mutation in BRCA1 or BRCA2. In The Bahamas $23 \%$ of women with breast cancer carry one of seven founder mutations in the BRCA1 or BRCA2 gene (BRCA1 :IVS13+1G>A， 4730insG, T5443G, IVS16+6T>C, 943ins10, 185delAG; BRCA2: 818delA, exons 8-9 del) (2). Incidentally BRCA1:IVS13+1G>A is the most common mutation found in African American family with hereditary breast and ovarian cancer syndrome (HBOC). This study sought to determine the frequency of BRCA1 or BRCA2 mutation in women with ovarian cancer or women with strong family history of breast or ovarian cancer.

Methodology: Women attending the Gynecology Oncology Clinic in the Oncology Center at Princess Margaret Hospital Nassau, Bahamas between March 2018 - March 2019 were offered genetic testing. The women were counseled and consented for genetic testing. Personal history of breast, endometrial or ovarian cancer was sought. Family history regarding any first-degree relative with breast or ovarian cancer was ascertained. Saliva samples underwent next generation sequencing (NGS) in a CLIA certified laboratory for a 30 gene panel linked to breast, ovarian and/or uterine cancer risks: $B R C A 1, B R C A 2$, Lynch genes, MLH1, MSH2, MSH6, PMS2, EPCAM, MUTYH, APC, STK11, PALB2, MITF, BAP1, CDKN2A, TP53, BMPR1A, SMAD4, POLD1, POLE1, CHEK2, PTEN, CDH1, BRIP1, CDK4, GREM1, RAD51C, RAD51D, PMS2, NBN and BARD1. Results and reports included presence or absence of deleterious mutations and variants of unknown significance (VUS).

Results: Between 03/2018-03/2019, 32 women were tested, diagnosed with ovarian cancer (19), endometrial cancer (9), breast cancer (2),breast and ovarian cancer (1) and women with a strong family history of breast/ ovarian cancer (2). The mean age at diagnosis was 60 years. The mean age of patients with BRCA1 was 62 and the mean age of patients with BRCA2 is 53 .
$21.9 \%$ had a deleterious mutation in BRCA: 5 in BRCA1 and 2 in BRCA2 $6 / 7$ women were diagnosed with ovarian cancer and 1 patient had both breast and ovarian cancer. 0/2 of the patients tested based on family history were positive. There were 5 patients with VUS: 2 PALB2, 1 BRCA1, 1-BRCA2 and 1-RAD51C.

\begin{tabular}{|l|l|}
\hline Age Distribution of Patients & Number of Patients \\
\hline $30-39$ & 2 \\
\hline $40-49$ & 6 \\
\hline $50-59$ & 8 \\
\hline $60-69$ & 8 \\
\hline 70 and Up & 8 \\
\hline
\end{tabular}

Frequency of Genetic Mutations

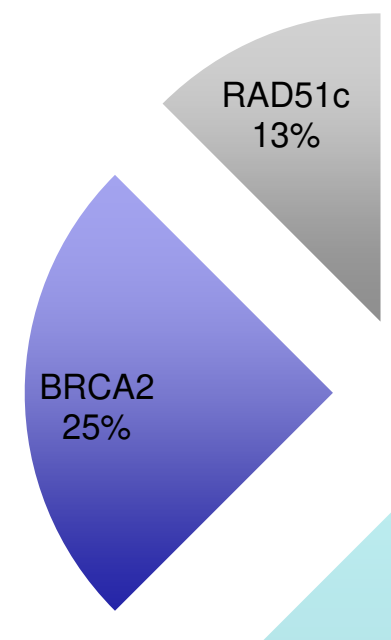

BRCA $62 \%$

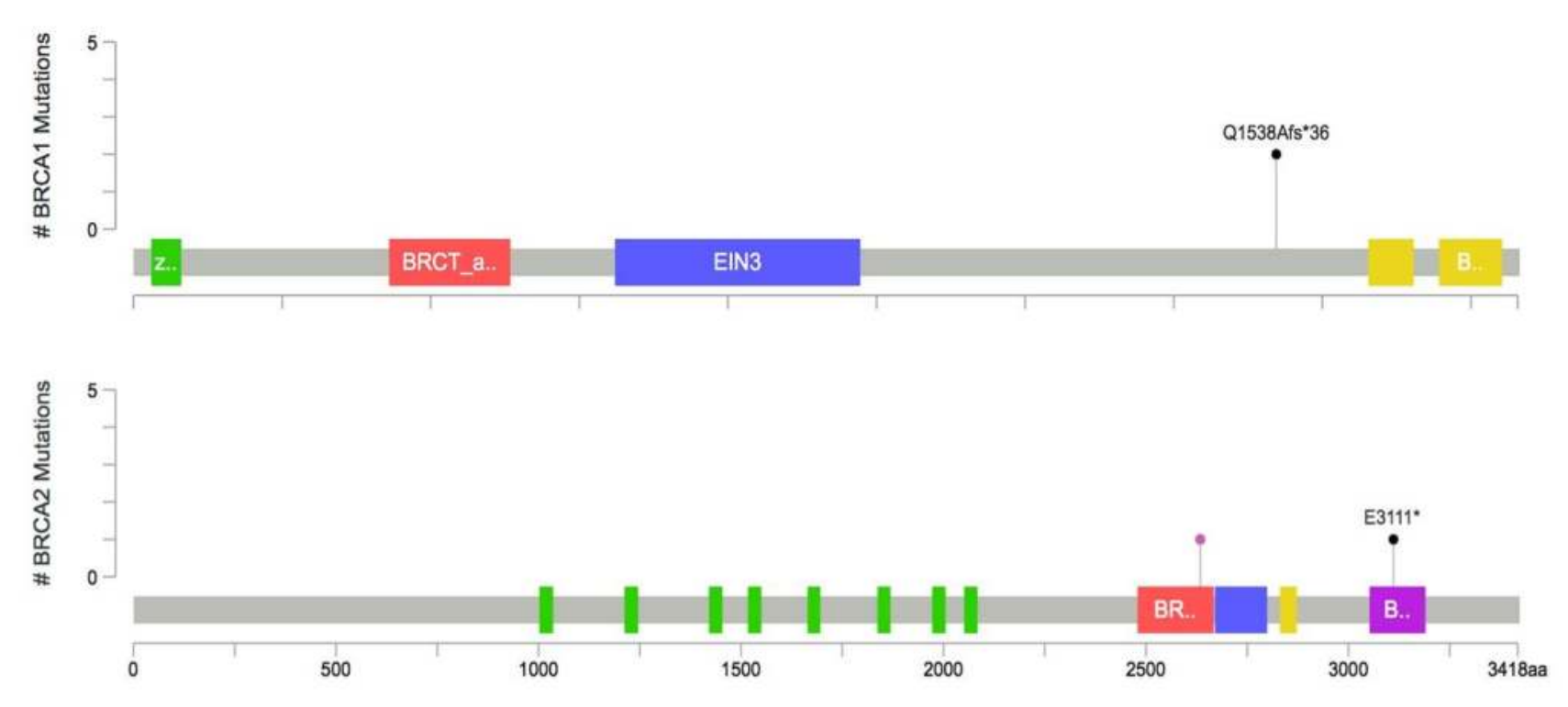

Conclusion: Genetic testing at point of care in the Bahamas is feasible and acceptable. Current NCCN guidelines recommend offering genetic testing for all women affected by ovarian cancer. In a setting with limited immunohistochemistry testing to confirm histological subtype it is prudent to follow such a recommendation. An Australian Ovarian Cancer Group report found the BRCA1/2 mutation in $14.1 \%$ of the patients studied (3). The rate of $21.9 \%$ in the limited population studied in the Bahamas is significant and warrants further studies. High prevalence of hereditary ovarian cancer is consistent with previous studies focused on breast cancer. These results highlight need for targeted therapy in Bahamian population of ovarian cancer patients. Genetic testing should be offered to all women with ovarian cancer as population-based testing for BRCA1/2 mutations in breast cancer detects a high proportion of carriers not identified by cancer family history-based testing. 